\title{
TRATAMIENTO DE LA DISFUNCIÓN ERÉCTIL TRAS PROSTATECTOMÍA RADICAL CON SILDENAFILO EN UN GRUPO NO SELECCIONADO DE PACIENTES
}

\author{
J. RUBIO BRIONES, I. IBORRA JUAN, R. DUMONT MARTÍNEZ, \\ J. CASANOVA RAMÓN-BORJA, J.L. MONRÓS LLISO, J.V. RICÓS TORRENT, \\ A. COLLADO SERRA, E. SOLSONA NARBÓN
}

Instituto Valenciano de Oncología. Valencia.

Actas Urol Esp. 28 (8): 567-574, 2004

\section{RESUMEN}

TRATAMIENTO DE LA DISFUNCIÓN ERÉCTIL TRAS PROSTATECTOMÍA RADICAL CON SILDENAFILO EN UN GRUPO NO SELECCIONADO DE PACIENTES

FUNDAMENTO: Conocer la disfunción eréctil (DE) tras prostatectomía radical en un grupo no seleccionado de pacientes y su respuesta al sildenafilo.

MÉTODOS: Incluimos a nuestros pacientes operados entre 1998 y 2001 . Los pacientes rellenaron una modificación del IIEF (mIIEF) antes de la PR. Probamos sildenafilo a dosis de 100 mg en 3 periodos diferentes: 3-6, 12 y 18-24 meses tras la PR. En caso de respuesta completa volvieron a rellenar el mIIEF.

RESULTADOS: La edad media fue de 62,8 años y el seguimiento medio de 31,7 meses. Sólo 111 (62\%) del total de pacientes operados quisieron tratamiento y tan solo 90 tomaron sildenafilo en el régimen propuesto; 27 (30\%), 18 (20\%) y 45 (50\%) tuvieron una respuesta completa, parcial o no tuvieron respuesta al sildenafilo respectivamente. El periodo más frecuente de respuesta completa fue entre los 18-24 meses. El mIIEF mostró 6,5 $\pm 5,7$ puntos menos respecto al mIIEF pre tratamiento. En el análisis univariado, la preservación de bandeletas (BNV), la presencia de respuesta parcial previa y la presencia de tumescencia previa se relacionaron significativamente con la respuesta completa; sin embargo, solo la tumescencia mantuvo su valor en el estudio multivariado.

CONCLUSIONES: El urólogo debe implicarse en la rehabilitación precoz de la FE incluso en los pacientes en los que no se ha podido realizar preservación de las BNV. Esta se debe intentar uni o bilateralmente siempre que el paciente desee preservar FE. La respuesta al sildenafilo es mejor a partir del primer año y mejor en aquellos que presentan tumescencia previa.

PALABRAS CLAVES: Impotencia. Prostatectomía radical. Sildenafilo.

\section{ABSTRACT}

TREATMENT OF POST RADICAL PROSTATECTOMY ERECTILE DYSFUNCTION WITH SILDENAFIL IN A NON-

\section{SELECTED GROUP OF PATIENTS}

OBJECTIVE: To evaluate erectile disfunction (ED) after radical prostatectomy in a non selected group of patients and their response to sildenafil.

METHODS: We included our patients who were operated on between 1998 and 2001. The patients filled in a modification of IIEF (mIIEF) before the RP operation. We tried sildenafil with doses of $100 \mathrm{mg}$ in 3 different periods: 3-6, 12 and 18-24 months after the RP operation. In the event of a complete response they filled in the mIIEF again.

RESULTS: The mean age of the patients was 62.8 years old and the mean observation period was 31.7 months. Only $111(62 \%)$ of the total number of patients operated on wanted treatment and only 90 took sildenafil in the proposed protocol; $27(30 \%), 18(20 \%)$ and $45(50 \%)$ patients had a complete response, a partial response or no response to the sildenafil respectively. The mIIEF showed $6.5 \pm 5.7$ points less than the mIIEF pre-treatment. In the univariate analysis, the preservation of bundles, the presence of a previous partial response and the presence of the previous tumescence were significantly related to the complete response; however, only tumescence kept its value in the multivariate study.

CONCLUSIONS: Urologists must involve themselves in the early rehabilitation of the erectile function even in those patients where preservation of the NVB was not possible. One must always try to preserve, uni or bilaterally, whenever the patient desires preservation of EF. The response to sildenafil is better after the first year and in patients who have previous tumescence. 
$\mathrm{S}_{\mathrm{a}}^{\mathrm{e}}$ han publicado resultados controvertidos acerca de la importancia de la función eréctil en la calidad de vida de los pacientes tras la prostatectomía radical (PR). Existen estudios que muestran tasas de satisfacción general con este procedimiento del $80 \%$ y la mayoría de los pacientes lo escogerían de nuevo independientemente de las tasas de disfunción eréctil $\mathrm{DE}^{1}$.

La mayoría de los españoles diagnosticados de cáncer de próstata escogen un tratamiento activo y rechazan la actitud expectante frente al mismo, por lo que la PR y la radioterapia externa son los tratamientos más escogidos ya que la braquiterapia y la prostatectomía radical laparoscópica están iniciando su andadura en nuestro centro. Sobre la impotencia tras PR se han barajado múltiples factores a tener en cuenta; edad, función eréctil ( $\mathrm{FE})$ previa, raza, factores clínico-patológicos y tiempo entre el tratamiento y la valoración de los resultados. Existen publicados resultados controvertidos sobre la preservación de la $\mathrm{FE}$ tras PR con conservación de BNV (Bandeletas Neuro Vasculares, cifrándose $33-80 \%$, 0-80\% y 0-20\% para la preservación bilateral, unilateral y la no preservación respectivamente ${ }^{2}$.

$\mathrm{El}$ sildenafilo es una de las drogas más utilizada para la DE. Ha mostrado su eficacia en DE tras PR en $43-80 \% \%^{3,4}$. La experiencia diaria con estos pacientes muestra cuan irregular es la respuesta a este medicamento, existiendo pocos estudios acerca del tiempo de administración, dosificación y seguimiento de su uso tras PR. Nuestro principal objetivo es saber cuándo está más indicado usar sildenafilo entre nuestros pacientes; además, conocer factores pronósticos en pacientes prostatectomizados que pudieran influir en la respuesta a esta droga.

\section{MATERIAL Y MÉTODOS}

Asumimos la definición de impotencia consensuada por el National Institutes of Health Consensus on Erectile Dysfunction como la imposibilidad de alcanzar y mantener una erección peneana suficiente para permitir una relación sexual satisfactoria ${ }^{5}$.

Seleccionamos los pacientes operados en nuestra Institución entre 1998 y 2000 que estaban dispuestos a rellenar cuestionarios sobre DE. No incluimos los pacientes que recibieron tratamiento hormonal neoadyuvante cuando eran remitidos de otros centros ni los que recibieron tratamiento adyuvante por presentar adenopatías positivas.

Usamos el International Index of Erectile Function (IIEF) como cuestionario por su sensibilidad y especificidad para valorar la función eréctil $^{6}$. Eliminamos las preguntas número 2, 4, 9, 13 y 14 del IIEF original, por lo que 4 de sus dominios de respuesta fueron analizados, a saber: función eréctil con las 1, 3, 5 y 15, función orgásmica con la pregunta 10 , deseo sexual con las preguntas 11 y 12 y la satisfacción con las relaciones con las preguntas 6,7 y 8 . En la Tabla 1 describimos nuestro score para los diferentes dominios de este IIEF modificado (mIIEF).

Tabla 1

Cuestionario IIEF modificado

\begin{tabular}{lcccc}
\hline & Severa & Moderada & Leve & No disfunción \\
\hline $\begin{array}{l}\text { Función } \\
\text { eréctil }\end{array}$ & $4-6$ & $7-10$ & $11-16$ & $17-20$ \\
$\begin{array}{l}\text { Función } \\
\text { orgásmica }\end{array}$ & 1 & $2-3$ & 4 & 5 \\
$\begin{array}{l}\text { Deseo } \\
\text { sexual }\end{array}$ & $1-2$ & $3-5$ & $6-8$ & $9-10$ \\
$\begin{array}{l}\text { Satisfacción } \\
\text { coital } \\
\text { mIIEF total }\end{array}$ & $1-3$ & $4-7$ & $8-11$ & $12-15$ \\
\hline
\end{tabular}

Nuestra intención fue determinar la situación real de nuestros pacientes acerca de su función eréctil; por lo tanto nuestro protocolo de rehabilitación de la función eréctil no excluyó ninguna edad y los pacientes eran libres de iniciar su rehabilitación en el momento que deseaban hacerlo por su estado de ánimo frente al trauma de la enfermedad y la cirugía, debido a que muchas veces prefieren posponer el tratamiento de la DE hasta que consiguen una continencia completa o constatan la buena evolución de su enfermedad oncológica. Todos rellenaron un mIIEF previamente a la PR. Si deseaban tratamiento de la DE y no existían contraindicaciones, les ofertábamos inyecciones intracavernosas (IIC) de Prostagladina E1 de forma precoz en los tres primeros meses tras PR, recomendando 2 por semana. Si no existían contraindicaciones absolutas (nitratos) o relativas (cardiopatía severa) y 
no habian requerido tratamiento hormonal por progresión de la enfermedad, e independientemente de la respuesta a la IIC, les ofrecíamos entre el $3^{\text {er }}$ y $6^{\circ}$ mes postoperatorio la primera prueba terapéutica con sildenafilo, que consistía en 4 tomas independientes de $100 \mathrm{mg}$ en un mes, resaltando las recomendaciones para su uso, haciendo hincapié en la existencia de deseo sexual y de evitar los periodos pospandriales.

La respuesta fue valorada de forma subjetiva (sin cuestionario) siempre por el mismo urólogo (JR), clasificándola en respuesta completa (potencia suficiente para mantener una relación satisfactoria según el paciente), en cuyo caso se les entregaba un segundo mIIEF para su objetivación y cuantificación, repuesta parcial (aumento de la tumescencia peneana insuficiente para mantener una relación) o no respuesta, obviando el cuestionario en estas dos últimas posibilidades por razones éticas. En caso de no respuesta los pacientes seguían con IIC. En caso de respuesta parcial podían probar el uso conjunto de sildenafilo $100 \mathrm{mg}$ más dispositivo de vacío (vacuum). Alrededor del año postratamiento, se les ofrecía a los pacientes una segunda prueba terapéutica con sildenafilo $100 \mathrm{mg} \mathrm{x}$ 4 tomas, valorando los resultados de la misma forma que en la primera. La tercera prueba terapéutica se realizaba entre los 18 y 24 meses postoperatorios. A ningún paciente se le planteó prótesis peneana antes de los 2 años de seguimiento.

En casos de respuesta completa, probábamos a reducir la dosis a $50 \mathrm{mg}$ a los 3 meses de la respuesta completa, para intentar reducir a $25 \mathrm{mg}$ a los 6 meses y probar al abandono de la medicación tras 9-12 meses de respuesta completa a una dosificación decreciente; lógicamente, en caso de empeoramiento en la respuesta, recomendábamos retomar la dosificación máxima de $100 \mathrm{mg}$. Nuestro protocolo de rehabilitación queda reflejado en la Figura 1.

Los datos fueron tratados mediante la versión 10.0 del SPSS para Windows (SPSS Inc., Illinois, U.S.A.), utilizando los test de Chi-cuadrado y de Fisher para el análisis univariado y la regresión logística multinominal para el estudio multivariado. El nivel considerado estadísticamente significativo fue 0,05 .

\section{RESULTADOS}

En el periodo escogido se operaron 179 pacientes. La edad media de los mismos fue $62,8 \pm 6,1$ años (rango 45-74). El seguimiento medio ha sido de 31,7 meses (rango 2-65). De ese grupo, 162 eran potentes antes de la PR y sólo 111 (62\%) de ellos mostraron interés en la recuperación de la función eréctil tras la cirugía. Este subgrupo de 111 pacientes tenía una edad media de 60,9 $\pm 6,01$ años y su mIIEF no mostraba disfunción eréctil (Tabla 1). Dos y cinco pacientes tenían contraindicaciones absolutas o relativas al sildenafilo respectivamente, en 7 casos fue necesaria la hormonoterapia adyuvante y otros 7 no quisieron tomar sildenafilo por su coste o por miedo a su uso, por lo que finalmente el grupo de estudio que se acogió en algún momento al algoritmo propuesto en la Figura 1 fue de 90 pacientes.

La libertad del momento de adhesión al protocolo de rehabilitación explica que 51, 63 y 44 tomaran sildenafilo en los 3 momentos propuestos respectivamente. Sólo 14 pacientes realizaron las 3 pruebas terapéuticas. En total, cuarenta y cinco pacientes no respondieron al sildenafilo (50\%), 18 (20\%) solo alcanzaron una respuesta parcial y 27 (30\%) consiguieron una respuesta completa. La respuesta completa fue más frecuente en la tercera prueba terapéutica (18-24 meses) con 16 pacientes, seguido de la segunda (12 meses) con 10 pacientes y solo uno respondió a los 6 meses.

Los respondedores completos fueron seguidos una media de 32 meses (14-58 de rango). La calificación total de sus respuestas en el mIIEF mostró $6,5 \pm 5,7$ puntos menos comparada con su mIIEF inicial; centrándose en el dominio de la función eréctil, este descenso fue cuantificado en $3,5 \pm 3,8$ también. Ocho pacientes pudieron abandonar el sildenafilo por completo y seguir manteniendo relaciones; 7 redujeron la dosis a $50 \mathrm{mg}, 11$ necesitaron de 100 mg para seguir manteniendo relaciones y uno tuvo que abandonarlo por cardiopatía sobrevenida.

De los 162 pacientes operados siendo potentes, cinco $(3,08 \%)$ recuperaron su potencia $\sin$ medicación a una media de 20 meses de seguimiento; dos de ellos espontáneamente y 3 tras 


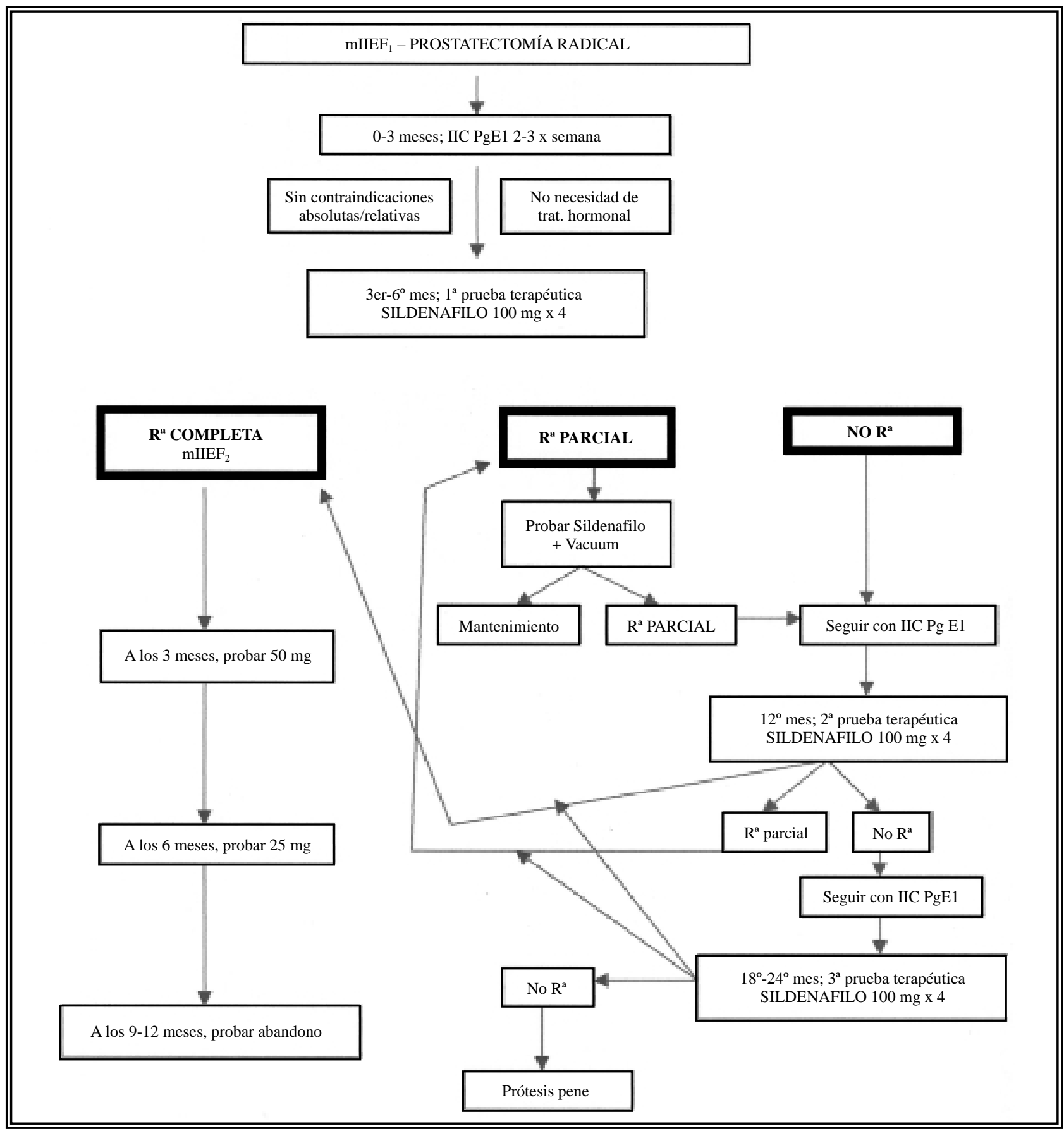

FIGURA 1. Algoritmo que muestra el protocolo de actuación utilizado para la rehabilitación de la función eréctil entre nuestros pacientes. (mIIEF; cuestionario IIEF, IIC Pg E1; inyecciones intracavernosas de prostaglandina E1, $R^{a}$; respuesta.

haber abandonado el protocolo de rehabilitación. Sumando a estos 5 pacientes los 27 respondedores completos, y excluyendo los 7 pacientes que requirieron hormonoterapia adyuvante, alcanzamos una tasa de recuperación de la potencia de $20,6 \%$ tras PR en nuestro grupo no seleccionado de pacientes.
Al final del estudio, 69 pacientes no seguían tomando sildenafilo por diversas razones (Tabla 2), sólo 4 por efectos adversos. Los dispositivos de vacío fueron utilizados por 3 pacientes, siempre asociados a sildenafilo en casos de respuesta parcial a éste, y sólo dos pacientes solicitaron la colocación de prótesis peneana. 
Tabla 2

Función eréctil final entre los 90 pacientes sujetos del estudio

\begin{tabular}{lc}
\hline Estado final (Fc. réctil) & № (\%) \\
\hline Potentes usando sildenafilo 100 mg & $11(12,2)$ \\
Potentes usando sildenafilo 25-50 mg & $7(7,7)$ \\
Potentes (sin tratamiento) & $11(12,2)$ \\
Usando IIC PgE1 & $18(20)$ \\
Combinando sildenafilo + vacuum & $3(3,3)$ \\
Prótesis peneana & $2(2,2)$ \\
Sin deseo de seguir tratamiento de DE & $25(27,7)$ \\
Abandono por efectos secundarios & $4(4,4)$ \\
Necesidad de hormonoterapia por progresión & $7(7,7)$ \\
Abandono por enfermedades concomitantes & $2(2,2)$ \\
\hline
\end{tabular}

IIC: inyecciones intracavernosas. PgE1: prostaglandina E1. DE: disfunción eréctil.

Para el análisis estadístico asumimos como respuesta tan sólo la respuesta completa, agrupando las respuestas parciales junto con las no respuestas. Distintos factores fueron calculados en relación con la respuesta y se muestran en la Tabla 3. Subrayar que tan solo 13 pacientes completaron un régimen de IIC de PGE1 precoz, de los cuales solo 3 tuvieron respuesta completa posteriormente al sildenafilo. Curiosamente, se objetivó una respuesta inversamente proporcional al uso de IIC de PgE1 en algún momento del protocolo de rehabilitación y la respuesta completa al sildenafilo, ya que 22 de los 27 respondedores completos nunca usaron PgE1. En este grupo no seleccionado de pacientes, se realizó preservación de las bandeletas neurovasculares en 14 pacientes $(12,6 \%), 8$ bilateral y 6 unilateral.

Por grupos etarios no se observaron diferencias en número de respuestas completas; $30 \%$ de los pacientes por debajo de los 55 años, 31,7\% para pacientes entre 56 y 65 años y $28,6 \%$ tras los 65 años.

En el análisis multivariado, la constatación de tumescencia espontánea fue el único factor pronóstico relacionado con la posibilidad de respuesta completa, con mayor poder estadístico que la preservación de bandeletas y la respuesta parcial previa al sildenafilo (Tabla 4). Resaltar que de los 34 pacientes en los que no se preservaron bandeletas y que presentaron en algún momento tumescencia peneana espontánea el $50 \%$ (17 pacientes) tuvieron una respuesta completa a $100 \mathrm{mg}$ de sildenafilo.

\section{DISCUSIÓN}

El cuestionario IIEF se ha mostrado como una excelente arma diagnóstica para medir la función eréctil ${ }^{6}$, pero fue originalmente diseñado para pacientes no prostatectomizados; ello conlleva que,

Tabla 3

Análisis univariado para la respuesta completa

\begin{tabular}{|c|c|c|c|c|c|}
\hline & No $r$ & sta & Respues & npleta & \\
\hline & Mediana & Rango & Mediana & Rango & \\
\hline Edad media & 60,97 & $45-73$ & 60,78 & $50-68$ & $\mathrm{P}=0,686$ (Anova) \\
\hline mIIEF total & 42,4 & $23-50$ & 43,3 & $38-47$ & $\mathrm{P}=0,557$ (Anova) \\
\hline & No (\%) & Si (\%) & No (\%) & Si (\%) & \\
\hline $\begin{array}{l}\text { Preservación } \\
\text { de BNV }\end{array}$ & $58(76,3)$ & $5(35,7)$ & $18(23,7)$ & $9(64,3)$ & $\mathrm{P}=0,002$ (Pearson)* \\
\hline $\begin{array}{l}\text { IIC de PgE } 1 \\
\text { precoz }\end{array}$ & $53(68,8)$ & $10(76,9)$ & $24(31,2)$ & $3(23,1)$ & $\mathrm{P}=0,556$ (Pearson) \\
\hline $\begin{array}{l}\text { IIC de PgE1 } \\
\text { seguimiento }\end{array}$ & $25(53,2)$ & $38(88,4)$ & $22(46,8)$ & $5(11,6)$ & $\mathrm{P}=0,000$ (Pearson) \\
\hline $\begin{array}{l}\text { Tumescencia } \\
\text { espontánea }\end{array}$ & $37(97,4)$ & $21(44,7)$ & $1(2,6)$ & $26(55,3)$ & $\mathrm{P}=0,000$ (Pearson) \\
\hline $\begin{array}{l}\text { Respuesta } \\
\text { parcial previa }\end{array}$ & $25(86,2)$ & $19(52,8)$ & $4(13,8)$ & $17(47,2)$ & $\mathrm{P}=0,004$ (Pearson) \\
\hline
\end{tabular}

(*). Calculado agrupando los casos de preservación uni y bilateral, aunque individualmente mantenía su significación 
Tabla 4

Análisis multivariado de los factores pronósticos para la respuesta completa

\begin{tabular}{lccccc}
\hline Variable & DF & X2/ Wald & P & Exp(B) & 95\% CI for Exp(B) \\
\hline $\begin{array}{l}\text { Preservación } \\
\text { BNV }\end{array}$ & 0,741 & 0,578 & 0,447 & 1,756 & $0,411 / 7,506$ \\
Tumescencia & 1,099 & 6,445 & 0,011 & $6,139 \mathrm{E}-02$ & $7,120 \mathrm{E}-=3 / 0,529$ \\
$\begin{array}{l}\text { Respuesta parcial } \\
\text { previa }\end{array}$ & 0,711 & 2,108 & 0,147 & 0,356 & $8,846 \mathrm{E}-02 / 1,435$ \\
\hline
\end{tabular}

por ejemplo, la pregunta número 9, que versa sobre el eyaculado, sea improcedente en los pacientes operados por cáncer de próstata. Cuando inicialmente pasamos el cuestionario original a nuestros pacientes, observamos que respondían más fácilmente a las preguntas 3 y 5 que a la 2 y la 4 , por lo que debido a su similitud excluimos las dos últimas. Finalmente, observamos que entre la población española operada mediante PR, su satisfacción general en cuanto a su vida sexual depende más del control de la enfermedad que de la función eréctil por sí misma, por lo que eliminamos las preguntas 13 y 14 para centrarnos en la respuesta objetiva al sildenafilo. Por estas razones y por la solicitud concomitante de otros cuestionarios (incontinencia, etc.) nos llevaron a reducir las originales 15 preguntas del IIEF a las 10 que hemos utilizado.

Se ha recomendado en la literatura la prevención de la disfunción eréctil tras PR con el uso de dispositivos de vacío o prostaglandina E1 precozmente en un intento de conservar la elasticidad y preservar la oxigenación del tejido ${ }^{7}$, y además recientemente el sildenafilo se ha propuesto como útil con este fin ${ }^{8}$. En nuestro protocolo no ofrecimos el sildenafilo nocturno profiláctico, pero sí el uso precoz de IIC de PgE1, recomendando 2 por semana. Sin embargo, hemos constatado la dificultad en inducir este tipo de regímenes en pacientes preocupados por su incontinencia y sobre todo en su enfermedad oncológica. No podemos concluir nada pues sobre la utilidad de la PgE1 precoz, debido al escaso número de pacientes que la usaron. Sin embargo, sî que parece que su práctica produce un efecto psicológico positivo al involucrar a los pacientes inicialmente en la recuperación de su función eréctil.
Una vez constatan nuestros pacientes la buena evolución de la enfermedad aceptan más fácilmente el uso de IIC si no responden al sildenafilo; de hecho el $20 \%$ de ellos prefirieron la PgE1 como tratamiento de su disfunción eréctil. Aunque su tasa de respuesta completa es alta (alrededor del 85\%), entre 40-58\% de los pacientes la abandonan por dolor, problemas con la inyección o fibrosis peneana a los 2 años de seguimiento. La inesperada relación inversa entre el uso en algún momento del protocolo de rehabilitación de IIC de PgE1 y la respuesta completa al sildenafilo en nuestra serie podría explicarse por la fibrosis que puedan inducir, pero probablemente se debe a que muchos de los no respondedores en la primera prueba terapéutica con sildenafilo que obtuvieron buena respuesta a las inyecciones, no vuelven a probar el sildenafilo ni en la segunda ni en la tercera pruebas terapéuticas.

Un estudio de la Cleveland Clinic muestra que el uso de sildenafilo a los 18 meses de la PR ofrece unas respuestas completas del 71,7\%, 50\% y $15,4 \%$ tras preservación bilateral, unilateral y no preservación de bandeletas respectivamente ${ }^{9}$. Zagaja y cols. apuntaron importantes diferencias no solo en la preservación, sino en la edad para la tasa de respuesta al sildenafilo ${ }^{3}$ en cirugías conservadoras, aunque no mostraron respuesta en cirugías no conservadoras.

Aunque en la mayoría de nuestros pacientes en el periodo de estudio no preservamos las BNV, creemos que éste es un factor importante en la recuperación de la potencia, aunque subrayamos la importancia de la presencia de tumescencia espontánea, incluso sin haber preservado las $\mathrm{BNV}$, como buen factor pronóstico para ese fin, por lo que es importante persuadir a los pacientes a un tratamiento activo para la recuperación 
de la potencia. Este hecho puede explicarse por cierto tipo de recuperación neurológica paralelo a la recuperación de la información nociceptiva causada por la disección quirúrgica y el proceso de cicatrización que se han propuesto como factores etiopatológicos de la $\mathrm{DE}$ tras $\mathrm{PR}^{10}$. Además, el hecho que durante los últimos años hayan aumentado el número de tumores organoconfinados (60-80\%) junto con nuestra mayor experiencia en PR (más de 700 procedimientos) nos han empujado a intentar preservar BNV más frecuentemente. Existen datos evidentes en la literatura mostrando la posibilidad de la preservación de $\mathrm{BNV}$, su relación con el estadio patológico, con la experiencia del urólogo y con la casuística del centro $^{11}$. Recientemente, se ha publicado en casos de preservación de BNV, que la administración nocturna de sildenafilo adelanta el retorno a erecciones espontáneas al año de seguimiento ${ }^{8}$, por lo que esto se podría plantear como una opción de momento en investigación clínica en ese tipo de pacientes, aunque habría que valorar posteriormente el alto coste de ese régimen.

Coincidimos con publicaciones previas en la ineficacia del sildenafilo en los 9 primeros meses post- $\mathrm{PR}^{2}$, debido probablemente a la neuropraxia, y ello coincide con la indicación de la rehabilitación precoz, o más bien profilaxis de la disfunción eréctil, llevada a cabo con IIC o vacuum. Si excluimos en nuestra serie aquellos pacientes que realizaron la primera prueba terapéutica y el único respondedor en ese periodo, observamos que $34,2 \%$ de los pacientes que lo usan tras un año de seguimiento, responden de forma completa al sildenafilo, $38,5 \%$ al año de la prostatectomía y $61,5 \%$ tras $18-24$ meses desde la cirugía.

Siguiendo nuestro protocolo de rehabilitación, once pacientes recuperaron erecciones espontáneas $(12,2 \%$ de los pacientes tratados), mientras que consiguieron erecciones mediadas por sildenafilo o IIC de PgE 1 otros 18 pacientes (20\% de los tratados) en cada grupo. Por lo tanto, la posibilidad que un paciente que siga este protocolo recupere la erección espontánea es del $12 \%$ y de forma asistida en un $40 \%$. Pensamos que es primordial en estos pacientes explicarles el uso correcto del sildenafilo o drogas similares. Se ha mostrado de manera exhaustiva por el estudio EDEM las dificultades del estudio y tratamiento de la DE en España ${ }^{12}$. Por lo tanto, el nivel de implicación del urólogo en la recuperación de la función eréctil es crucial ${ }^{13}$. Tras la PR, es necesario comenzar usando las dosis máximas de las 3 drogas en el mercado, sildenafil, tadalafilo y vardenafilo $^{14}$.

Además, creemos necesario que tanto el paciente como su pareja se vean involucrados en el tratamiento de la disfunción eréctil lo antes posible, por lo que el régimen precoz con IIC, independientemente de su resultado profiláctico, tiente un efecto psicológico muy positivo en la pareja. La empatía de la pareja con un urólogo motivado es muy importante para la recuperación de la potencia, pues da pie a la utilización de todos los recursos terapéuticos de una forma racional y progresiva sin caer en el desánimo que pueda producir la no respuesta a un tratamiento concreto. Esto, sumado al objetivo de aumentar las tasas de potencia post PR realizando más cirugías conservadoras de BNV en centros de referencia debería incrementar dichas tasas en nuestro país, haciendo esta cirugía más competitiva con otras modalidades de tratamiento también desde el punto de función sexual. En un futuro próximo conoceremos los resultados de las grandes series de PR Laparoscópica, que por la magnificación del campo quirúrgico y la precisión en su disección, es de esperar que ofrezcan mejores resultados en cuanto a preservación de BNV y por tanto en la recuperación de la función eréctil.

\section{CONCLUSIONES}

Abogamos por la necesidad de la implicación del urólogo en la recuperación de la disfunción eréctil antes y después de la PR. Tras analizar nuestros resultados, estamos intentando preservar más BNV cuando ello es posible y los pacientes desean preservar su función eréctil. Debido a su práctica generalizada, los pacientes con deseo de conservar su potencia deberían ser referidos a centros de referencia. Incluso en los pacientes en los que no es posible la preservación de BNV, vale la pena implementar un protocolo de rehabilitación con IIC durante el primer año y probar sildenafilo $100 \mathrm{mg}$ a partir del mismo, esperando mejores resultados en aquellos que presentan tumescencia previa. 
Agradecimientos: al Dr. Eduardo Nagore por su inestimable ayuda en el análisis estadístico de los datos.

\section{REFERENCIAS}

1. Kao TC, Cruess DF, Garner D, Foley J, Seay T, Fiderichs P et al. Multicenter patient self-reporting questionnnaire on impotence. Comment J Urol 2000;163:870-878.

2. Carson CC, Burnett AL, Levine LA, Nehra A. The efficacy of sildenafil citrate (Viagra) in clinical populations. An update. Urology 2002;60(Supplement 2B):12-27.

3. Zagaja GP, Mhoon DA, Aikens JE, Brendler CB. Sildenafil in the treatment of erectile dysfunction after radical prostatectomy. Urology 2000;56(4):631-634.

4. Zippe C, Kdeia AW, Kdeia K et al. Treatment of erectile dysfunction following radical prostatectomy with sildenafil citrate (Viagra). Urology 1998;52:963-966.

5. NIH consensus development on impotence. JAMA 1993; 270:83.

6. Rosen RC, Riley A, Wagner G, Osterloh IH, Kipratrick J, Mishra A. The international index of erectile function (IIEF): a multidimensional scale for assessment of erectile dysfunction. Urology 1997;49(6):822-830.

7. Montorsi F, Guazzoni G, Strambi LF, Da Pozzo LF, Nava L, Barbieri L, Rigatti P, Pizzini G, Miani A. Recovery of spontaneous erectile function after nerve-sparing radical retropubical prostatectomy with and without early intracavernous injections of alprostadil: results of a prospective, randomized trial. J Urol 1997;158:1408-1410.

8. Padma-Nathan H, Mc Coullough AR, Giuliano F, Toler SM, Wohlhuter C, Shpilsky AB. Postoperative nightly administration of sildenafil citrate significantly improves the return of normal spontaneous erectile function after bilateral nerve-sparing radical prostatectomy. (Abstract 1402). J Urol 2003; 169(4):375.
9. Zippe CD, Jhaveri FM, Klein EA, Kdeia S, Pasqualotto FF, Kdeia A, Agarwal A, Montague AK, Lakin MM. Role of viagra after radical prostatectomy. Urology 2000;55(2):241247.

10. Mauleman EJH, Mulders PFA. Erectile function after radical prostatectomy: a review. Eur Urol 2002;43:95100.

11. Begg CB, Rideel ER, Bach PB, Kattan MW, Schag D, Warren JL, Scardino PT. Variations in morbidity after radical prostatectomy. New England J MDE 2002;346:11381143.

12. Martín Morales A, Sánchez Cruz JJ, Sáenz De Tejada I, Rodríguez Vela L, Jiménez Cruz JF, Burgos Rodríguez R. Prevalence and independent risk factors for erectile dysfunction in Spain: results of the Epidemiologia de la Disfunción Eréctil Masculina Study. J Urol 2001;166(2): 569-575.

13. McCulloough AR, Barada JH, Fawzy A, Guay AT, Hatzichristou D. Achieving treatment optimization with sildenafil citrate (Viagra) in patients with erectile dysfunction. Urology 2000;60(Supplement 2B):28-38.

14. Brock G, Taylor T, Seger M, Vardenafil Prospect Group. Efficacy and tolerability of vardenafil in men with erectile dysfunction following radical prostatectomy. (Abstract 598) Eur Urol 2002;1(suppl 1):152.

\author{
Dr. J. Rubio Briones \\ Instituto Valenciano de Oncología \\ C/ Profesor Beltrán Báguena, 8 \\ 46009 Valencia
}

(Trabajo recibido el 1 diciembre de 2003) 